\title{
Behaviour of Thin Magnetic Films at Low Temperatures
}

\author{
Vjekoslav Sajertt ${ }^{1} *$, Dušan Popov², Ljiljana Mašković ${ }^{3}$, and Bratislav Tošić ${ }^{4}$ \\ ${ }^{1}$ Technical Faculty "Mihajlo Pupin" Zrenjanin, Serbia \\ ${ }^{2}$ Universitatea "Politechnica," Timisoara, Romania \\ ${ }^{3}$ Police Academy, Belgrade, Serbia \\ ${ }^{4}$ Vojvodina Academy of Science and Arts, Novi Sad, Serbia
}

\begin{abstract}
The problem of the analysis of low temperature magnetization is exposed in detail. The application of exact Bose representation of spin operators to the bulk ferromagnets is justified. By means of this representation the magnetization of ultrathin films was analysed. It turned out that thin films magnetization contains exponentially small terms characterized by Dyson's functions even in harmonical approximation. The main conclusions of this work are that magnetic lattice of thin film is more rigid than the macroscopic lattice and that the autoreduction process (the three layer film divides onto three two layer subfilms) takes place in the film.
\end{abstract}

Keywords: Ultrathin Magnetic Films, Low Temperature Magnetization, Exact Bose Representation, Two Small Parameters Autoreduction, Qualitative Difference Nanoand Macrostructures.

\section{INTRODUCTION}

The Hamiltonian of Heisenberg model of isotropic ferromagnet is given by:

$$
\begin{aligned}
H= & S J_{0} \sum_{\vec{n}}\left(S-S_{\vec{n}}^{z}\right)-\frac{1}{2} \sum_{\vec{n}, \vec{m}} I_{\vec{n}, \vec{m}} S_{\vec{n}}^{-} S_{\vec{n}}^{+} \\
& -\frac{1}{2} \sum_{\vec{n}, \vec{m}} I_{\vec{n}, \vec{m}}\left(S-S_{\vec{n}}^{z}\right)\left(S-S_{\vec{m}}^{z}\right)
\end{aligned}
$$

In this formula $S^{+}=S^{x}+i S^{y}, S^{+}=S^{x}+i S^{y}$ are spin operators, $I_{\vec{n}, \vec{m}}$ are exchange integrals and $J_{0}=\sum_{\vec{l}} I_{\vec{l}}$. The spin operators obey the following commutation relations:

$$
\left[S^{+}, S^{-}\right]=2 S^{z} ; \quad\left[S^{+}, S^{z}\right]=-S^{+} ; \quad\left[S^{-}, S^{z}\right]=S^{-}
$$

It is obvious from (2) that spin commutation relations differ from Bozon commutation relations as well as from fermion commutation rules. This fact caused many discussions, which were lasting for about thirty years. The mentioned discussions were concerned mainly with attempts of creating the real Bozon picture of low temperature ferromagnet.

The first theory of low temperature magnetism was given by Bloch. ${ }^{1,2} \mathrm{He}$ rejected the last term of spin Hamiltonian (1), which represents dynamical interaction

\footnotetext{
*Author to whom correspondence should be addressed.
}

of spin waves and used the following Bozon representation of spin operators

$$
S_{\vec{n}}^{+}=\sqrt{2 S} B_{\vec{n}} ; \quad S_{\vec{n}}^{-}=\sqrt{2 S} B_{\vec{n}}^{+} ; \quad S-S_{\vec{n}}^{z}=B_{\vec{n}}^{+} B_{\vec{n}}
$$

This Bloch's approximation is called approximate second quantization method, today. Since the operator $S-S^{z}$ takes finite set of values $0,1,2, \ldots, 2 S$ it is clear that this approximation becomes incorrect when the eigenvalues of Bozon occupation number operator $B^{+} B$ is higher than values $2 S$. In connection with this arose problem of nonphysical states, i.e., the states when $\left(B^{+} B\right)_{\text {eigen }}>2 S$.

The Hamiltonian (1) in Bloch's approximation has the following Bozon form:

$$
H=S J_{0} \sum_{\vec{n}} B_{\vec{n}}^{+} B_{\vec{n}}-S \sum_{\vec{n}, \vec{m}} I_{\vec{n}, \vec{m}} B_{\vec{n}}^{+} B_{\vec{m}}
$$

This Hamiltonian can be diagonalized by means of Fourier transformation of Bose operators $B_{\vec{n}}^{+}$and $B_{\vec{n}}$ and consequently, from diagonalized form can be found the dispersion law of spin waves. Using this dispersion law and the approximations (3) Bloch obtained the following expression for low temperature relative magnetization:

$$
\sigma=\frac{\left\langle S^{z}\right\rangle}{S}=1-\frac{1}{S} \zeta_{3 / 2} \tau^{3 / 2} ; \quad \tau=\frac{\Theta}{2 \pi I} ; \quad \Theta=k_{\mathrm{B}} T
$$

The Riemann's $\zeta$ function is given by:

$$
\zeta_{p}=\sum_{n=1}^{\infty} \frac{1}{n^{p}}
$$


The following step in low temperature analyses of ferromagnet was done by Holstein and Primakoff. ${ }^{3}$ They generalized Bloch's approximation using the following Bozon approximation of spin operators:

$$
\begin{gathered}
S^{+}=\sqrt{2 S} \sqrt{1-\frac{B^{+} B}{2 S}} B \\
S^{-}=\sqrt{2 S} B^{+} \sqrt{1-\frac{B^{+} B}{2 S}} \\
S^{z}=S-B^{+} B
\end{gathered}
$$

It is seen that for $S=1 / 2$ these formulas are valid if maximal eigenvalue of operator $B^{+} B$ is equal to unit. In accordance with this, square root $\sqrt{1-B^{+} B}$ transit into $1-B^{+} B$. Besides the upper Bozon representation of spin operators the dynamical interaction term from (1) was conserved in their picture. Using transition $\sqrt{1-B^{+} B} \rightarrow$ $1-B^{+} B$ for $S=1 / 2$ and expanding the square roots from (7) they obtained additional fourth order term in Bozon Hamiltonian that is known as kinematical interaction of spin waves because it arose due to the difference of spin and Bose commutation rules. As in the case of Bloch's model the problem of non-physical states remained in the Holstein Primakoff model, also. The Bozon Hamiltonian obtained in the described way was analysed by Green's function method and for relative magnetization of simple cubic ferromagnet with spin $S=1 / 2$ was obtained the formula:

$$
\begin{aligned}
\sigma=1 & -2 \zeta_{3 / 2} \tau^{3 / 2}-\frac{3 \pi}{2} \zeta_{5 / 2} \tau^{5 / 2}-\frac{33 \pi^{2}}{16} \zeta_{7 / 2} \tau^{7 / 2} \\
& -6 \pi \zeta_{3 / 2} \zeta_{5 / 2} \tau^{4}+O\left(\tau^{9 / 2}\right)
\end{aligned}
$$

The term proportional to $\tau^{4}$ is the consequence of spin waves interaction. All other terms in (8) correspond to harmonical Bloch's approximation. In Bloch's theory the Fourier transform of exchange integral $J_{\vec{k}}=\sum_{\vec{l}} I_{\vec{l}} e^{i \vec{k} \vec{l}}$ was expanded up to $|\vec{k}|^{2}$. The terms proportional to $\tau^{3 / 2}$ and $\tau^{7 / 2}$ in (8) are the consequence of expansion $J_{\vec{k}}$ up to $|\vec{k}|^{6}$. The result (8) for low temperature magnetization was considered as correct, but the suspicion remained: are non-physical states correctly eliminated?

The correct theory of low temperature magnetization, where the problem of non-physical states does not appear, is given by Dyson. ${ }^{4,5}$ Dyson has shown that the contributions of the states with two and more Bozons on the lattice site are exponentially small. Using this proof he obtained the same expression for low temperature magnetization as Holstein and Primakoff.

Using Dyson's theory Maleev ${ }^{6}$ formulated the following Bozon representation of spin operators:

$$
\begin{gathered}
S^{+}=\sqrt{2 S}\left(1-\frac{1}{2 S} B^{+} B\right) B \\
S^{-}=\sqrt{2 S} B^{+} \\
S^{z}=S-B^{+} B
\end{gathered}
$$

which leads to the result ( 8 ) for spin $S=1 / 2$. It is seen that the representation (9) is not hermitian. The expression for $S^{-}$is given on the basis of Dyson's proof that the contributions of two or more Bozons on the lattice site are negligible. The representation (9) is called Dyson Maleev representation.

Next step investigation of low temperature magnetization were analyses by means of spin Green's function.

The first attempt was done by Tyablikov. ${ }^{7}$ He analysed ferromagnet with spin $S=1 / 2$. The Hamiltonian of ferromagnet was expressed in terms of Pauli operators $P^{+}$and $P$ and the paulion Green's function $\left\langle\left\langle P_{\vec{n}}(t) \mid P_{\vec{m}}^{+}(0)\right\rangle\right\rangle \equiv$ $\theta(t)\left\langle\left[P_{\vec{n}}(t), P_{\vec{m}}^{+}(0)\right]\right\rangle$, where $\theta(t)$ is Heaviside step function, ${ }^{8}$ was calculated. The magnon concentrations were found with the help of spectral intensity of paulion Green's function. Substituting spin concentrations, calculated in this way, in the expression for relative magnetization Tyablikov obtained the correction of harmonical (Bloch's) magnetization, which is proportional to $\tau^{3}$ and positive. This correction is known in the literature as "spurious error."

In ferromagnet with $S>1 / 2$ the "spurious error" does not appear. It was shown in series of references ${ }^{9-17}$ etc. In mentioned works the system of $2 S$ Green's functions

$$
\begin{aligned}
\Gamma_{\vec{n}, \vec{m}}(t)=\theta(t)\left\langle\left[P_{\vec{n}}(t), P_{\vec{m}}^{+}(0)\right]\right\rangle \equiv & \left\langle\left\langle S^{+}\left(S^{z}\right)^{2 j-1} \mid S^{-}\right\rangle\right\rangle ; \\
& 2 j=1,2, \ldots, S
\end{aligned}
$$

was solved. It turned out that the first anharmonic correction of magnetization for spin $S>1 / 2$ was proportional to square of magnon concentrations ${ }^{7}$ (chap. 35.21, p. 265), i.e., proportional to $\tau^{9 / 2}$. For $S=1 / 2$ the first anharmonic correction was proportional to square of magnon concentration, i.e., proportional to $\tau^{3}$. It means that the problem of "spurious error" for $S=1 / 2$ was not eliminated.

Several years ago the problem of low temperature magnetization was treated by means of exact Bose representation. ${ }^{18}$ In this representation Pauli operators were represented by infinite Bose operators series. The commutation rules of these Bozon series in Bozon space were identical with Pauli commutation rules in Pauli operator space. In this way the problem of non-physical states was eliminated. In the work ${ }^{19}$ was obtained the correct expression for magnetization of antiferromagnet with spin $S=1 / 2$. In the work ${ }^{20}$ was pointed out that by the use of exact Bozon representation of Pauli operators the problem of "spurious error" could be successfully solved.

The magnetization of thin film will be analysed by means of exact Bozon representation. The reason for this is the fact that in thin films, as we shall see later, already in harmonic (Bloch's) approximation exponentially small terms appear. Due to this, the Dyson's proof that contributions of the states with two and more Bozons at one lattice site, is not applicable in the case of thin films, since this proof is related to anharmonic effect only. 
In the last time interest for magnetic problems increases, ${ }^{21-30}$ but nowhere the problem of broken symmetry structures is not in the first plan.

Since the whole further analysis will be done by means of exact Bose representation we shall prove in the next section that this representation leads to correct formula for low temperature magnetization in the case of macroscopic (bulk) structure.

In the third section we shall analyse low temperature behaviour of films in Bloch's approximation. It is necessary since some of harmonic formulas define the low temperature anharmonic corrections.

In the last section will be realised the main goal of this work, i.e., investigation influence of spin waves interaction to low temperature magnetization of magnetic film.

\section{LOW TEMPERATURE MAGNETIZATION IN IDEAL SIMPLE CUBIC STRUCTURE WITH SPIN $S=1 / 2$}

Since the exact Bose representation of spin operators for $S=1 / 2$ (Pauli operators) will be used in analysis of thin magnetic film, we shall prove in this section that its application leads to correct, Dyson's result. The Hamiltonian of this ferromagnet will be expressed in terms of Pauli operators which are connected with spin operators for $S=1 / 2$ by the following relations: $S^{+}=P, S^{-}=P^{+}$and $1 / 2-$ $S^{z}=P^{+} P$.

Consequently, the form of the Hamiltonian of this ferromagnet, written in the nearest neighbour's approximation is the following:

$$
\begin{array}{r}
H=\frac{1}{2} \sum_{\vec{n}}\left(I_{\vec{n}, \vec{n}+\vec{a}_{x}}+I_{\vec{n}, \vec{n}-\vec{a}_{x}}+I_{\vec{n}, \vec{n}+\vec{a}_{y}}+I_{\vec{n}, \vec{n}-\vec{a}_{y}}\right. \\
\left.+I_{\vec{n}, \vec{n}+\vec{a}_{z}}+I_{\vec{n}, \vec{n}-\vec{a}_{z}}\right) P_{\vec{n}}^{+} P_{\vec{n}} \\
-\frac{1}{2} \sum_{\vec{n}} P_{\vec{n}}^{+}\left(I_{\vec{n}, \vec{n}+\vec{a}_{x}} P_{\vec{n}+\vec{a}_{x}}+I_{\vec{n}, \vec{n}-\vec{a}_{x}} P_{\vec{n}-\vec{a}_{x}}+I_{\vec{n}, \vec{n}+\vec{a}_{y}} P_{\vec{n}+\vec{a}_{y}}\right. \\
\left.\quad+I_{\vec{n}, \vec{n}-\vec{a}_{y}} P_{\vec{n}-\vec{a}_{y}}+I_{\vec{n}, \vec{n}+\vec{a}_{z}} P_{\vec{n}+\vec{a}_{z}}+I_{\vec{n}, \vec{n}-\vec{a}_{z}} P_{\vec{n}-\vec{a}_{z}}\right) \\
-\frac{1}{2} \sum_{\vec{n}} P_{\vec{n}}^{+} P_{\vec{n}}\left(I_{\vec{n}, \vec{n}+\vec{a}_{x}} P_{\vec{n}+\vec{a}_{x}}^{+} P_{\vec{n}+\vec{a}_{x}}+I_{\vec{n}, \vec{n}-\vec{a}_{x}} P_{\vec{n}-\vec{a}_{x}}^{+} P_{\vec{n}-\vec{a}_{x}}\right. \\
\quad+I_{\vec{n}, \vec{n}+\vec{a}_{y}} P_{\vec{n}+\vec{a}_{y}}^{+} P_{\vec{n}+\vec{a}_{y}}+I_{\vec{n}, \vec{n}-\vec{a}_{y}} P_{\vec{n}-\vec{a}_{y}}^{+} P_{\vec{n}-\vec{a}_{y}} \\
\left.\quad+I_{\vec{n}, \vec{n}+\vec{a}_{z}} P_{\vec{n}+\vec{a}_{z}}^{+} P_{\vec{n}+\vec{a}_{z}}+I_{\vec{n}, \vec{n}-\vec{a}_{z}} P_{\vec{n}+\vec{a}_{z}}^{+} P_{\vec{n}-\vec{a}_{z}}\right)
\end{array}
$$

where $\vec{a}_{x}, \vec{a}_{y}$ and $\vec{a}_{z}$ are connecting the nearest neighbours.

The analysis will be carried out by means of paulion Green's function

$$
\Gamma_{\vec{n}, \vec{m}}(t)=\theta(t)\left\langle\left[P_{\vec{n}}(t), P_{\vec{m}}^{+}(0)\right]\right\rangle \equiv\left\langle\left\langle P_{\vec{n}}(t) \mid P_{\vec{m}}^{+}(0)\right\rangle\right\rangle
$$

where $\theta(t)$ is Heaviside step-function.
Since we deal with ideal structure, the exchange integrals are equal everywhere, and therefore we shall take:

$$
I_{\vec{n}, \vec{n} \pm \vec{a}_{x}}=I_{\vec{n}, \vec{n} \pm \vec{a}_{y}}=I_{\vec{n}, \vec{n} \pm \vec{a}_{z}}=I
$$

Differentiating $\Gamma_{\vec{n}, \vec{m}}(t)$ with respect to $t$ and taking into account that $\dot{P}_{\vec{n}}=(1 /(i \hbar))\left[P_{\vec{n}}, H\right]$ we obtain the following equation

$$
\begin{array}{rl}
i \hbar \frac{d}{d t} \Gamma_{\vec{n}, \vec{m}}(t) & \\
=i \hbar \delta & (t) \delta_{\vec{n}, \vec{m}}\left(1-2\left\langle P_{\vec{n}}^{+} P_{\vec{n}}\right\rangle\right)+3 I \Gamma_{\vec{n}, \vec{m}}(t) \\
-\frac{1}{2} I & I \Gamma_{\vec{n}+\vec{a}_{x}, \vec{n}}(t)+\Gamma_{\vec{n}-\vec{a}_{x}, \vec{n}}(t)+\Gamma_{\vec{n}+\vec{a}_{y}, \vec{n}}(t) \\
& \left.+\Gamma_{\vec{n}-\vec{a}_{y}, \vec{n}}(t)+\Gamma_{\vec{n}+\vec{a}_{z}, \vec{n}}(t)+\Gamma_{\vec{n}-\vec{a}_{z}, \vec{n}}(t)\right) \\
+I & \left\langle\left\langle\left\langle P_{\vec{n}}^{+}(t) P_{\vec{n}}(t) P_{\vec{n}+\vec{a}_{x}}(t) \mid P_{\vec{m}}^{+}(0)\right\rangle\right\rangle\right. \\
& +\left\langle\left\langle P_{\vec{n}}^{+}(t) P_{\vec{n}}(t) P_{\vec{n}-\vec{a}_{x}}(t) \mid P_{\vec{m}}^{+}(0)\right\rangle\right\rangle \\
& +\left\langle\left\langle P_{\vec{n}}^{+}(t) P_{\vec{n}}(t) P_{\vec{n}+\vec{a}_{y}}(t) \mid P_{\vec{m}}^{+}(0)\right\rangle\right\rangle \\
& +\left\langle\left\langle P_{\vec{n}}^{+}(t) P_{\vec{n}}(t) P_{\vec{n}-\vec{a}_{y}}(t) \mid P_{\vec{m}}^{+}(0)\right\rangle\right\rangle \\
& +\left\langle\left\langle P_{\vec{n}}^{+}(t) P_{\vec{n}}(t) P_{\vec{n}+\vec{a}_{z}}(t) \mid P_{\vec{m}}^{+}(0)\right\rangle\right\rangle \\
& \left.+\left\langle\left\langle P_{\vec{n}}^{+}(t) P_{\vec{n}}(t) P_{\vec{n}-\vec{a}_{z}}(t) \mid P_{\vec{m}}^{+}(0)\right\rangle\right\rangle\right) \\
-I & \left\langle\left\langle\left\langle P_{\vec{n}+\vec{a}_{x}}^{+}(t) P_{\vec{n}+\vec{a}_{x}}(t) P_{\vec{n}}(t) \mid P_{\vec{m}}^{+}(0)\right\rangle\right\rangle\right. \\
& +\left\langle\left\langle P_{\vec{n}-\vec{a}_{x}}^{+}(t) P_{\vec{n}-\vec{a}_{x}}(t) P_{\vec{n}}(t) \mid P_{\vec{m}}^{+}(0)\right\rangle\right\rangle \\
& +\left\langle\left\langle P_{\vec{n}+\vec{a}_{y}}^{+}(t) P_{\vec{n}+\vec{a}_{y}}(t) P_{\vec{n}}(t) \mid P_{\vec{m}}^{+}(0)\right\rangle\right\rangle \\
& +\left\langle\left\langle\left\langle P_{\vec{n}-\vec{a}_{y}}^{+}(t) P_{\vec{n}-\vec{a}_{y}}(t) P_{\vec{n}}(t) \mid P_{\vec{m}}^{+}(0)\right\rangle\right\rangle\right. \\
& +\left\langle\left\langle\left\langle P_{\vec{n}+\vec{a}_{z}}^{+}(t) P_{\vec{n}+\vec{a}_{z}}(t) P_{\vec{n}}(t) \mid P_{\vec{m}}^{+}(0)\right\rangle\right\rangle\right. \\
& \left.+\left\langle\left\langle P_{\vec{n}-\vec{a}_{z}}^{+}(t) P_{\vec{n}-\vec{a}_{z}}(t) P_{\vec{n}}(t) \mid P_{\vec{m}}^{+}(0)\right\rangle\right\rangle\right)
\end{array}
$$

At this stage of the analysis we shall substitute Pauli operators $P$ with Bose operators $B$ using exact Bozon representation of Pauli operators:

$$
\begin{gathered}
P=\left[\sum_{\nu=0}^{\infty} \frac{(-2)^{\nu}}{(1+\nu) !} B^{+\nu} B^{\nu}\right]^{1 / 2} B \\
P^{+}=B^{+}\left[\sum_{\nu=0}^{\infty} \frac{(-2)^{\nu}}{(1+\nu) !} B^{+\nu} B^{\nu}\right]^{1 / 2} \\
P^{+} P=\sum_{\nu=0}^{\infty} \frac{(-2)^{\nu}}{(1+\nu) !}\left(B^{+}\right)^{\nu+1} B^{\nu+1}
\end{gathered}
$$

In low temperature range the upper representation can be used in the following approximation:

$$
\begin{aligned}
P_{\vec{n}} & =B_{\vec{n}}-B_{\vec{n}}^{+} B_{\vec{n}} B_{\vec{n}} \\
P_{\vec{n}}^{+} & =B_{\vec{n}}^{+}-B_{\vec{n}}^{+} B_{\vec{n}}^{+} B_{\vec{n}} \\
P_{\vec{n}}^{+} P_{\vec{n}} & =B_{\vec{n}}^{+} B_{\vec{n}}-\left(B_{\vec{n}}^{+}\right)^{2} B_{\vec{n}}^{2}
\end{aligned}
$$

Substitution (16)-(18) has to be done in the following way. In the Green's function $\Gamma$ must be used whole 
expressions on the right hand side of (16) and (17). In the higher order Green's functions Pauli operators may be substituted with Bose operators, i.e., $P \approx B$ and $P^{+} \approx B^{+}$. Finally in correlator may be taken $\left\langle P_{\vec{n}}^{+} P_{\vec{n}}\right\rangle \approx\left\langle B_{\vec{n}}^{+} B_{\vec{n}}\right\rangle$.

In the Eq. (14) expressed in terms of Bose operators, in described way, higher order Bozon Green's functions will be transformed by means of Wick's theorem. In such a way we obtain:

$$
\begin{aligned}
\left\langle\left\langle P_{\vec{n}}(t) \mid P_{\vec{m}}^{+}(0)\right\rangle\right\rangle \approx\langle & \mid\left(B_{\vec{n}}(t)-B_{\vec{n}}^{+}(t) B_{\vec{n}}(t) B_{\vec{n}}(t)\right) \\
\approx & \left.\left|\left\langle B_{\vec{m}}^{+}(0)-B_{\vec{n}}^{+}(0) B_{\vec{m}}^{+}(0) B_{\vec{n}}(0)\right)\right\rangle\right\rangle \\
& -\left\langle\left\langle B_{\vec{m}}^{+}(0)\right\rangle\right\rangle \\
& -\left\langle\left\langle B_{\vec{n}}(t) \mid B_{\vec{n}}(t) B_{\vec{n}}^{+}(0) B_{\vec{m}}^{+}(0) B_{\vec{n}}(0)\right\rangle\right\rangle \\
\approx & \left\langle\left\langle B_{\vec{n}}(t) \mid B_{\vec{m}}^{+}(0)\right\rangle\right\rangle \\
& -2\left\langle B_{\vec{n}}^{+} B_{\vec{n}}\right\rangle^{(0)}\left\langle\left\langle B_{\vec{n}}(t) \mid B_{\vec{m}}^{+}(0)\right\rangle\right\rangle \\
& -2\left\langle B_{\vec{m}}^{+} B_{\vec{m}}\right\rangle^{(0)}\left\langle\left\langle B_{\vec{n}}(t) \mid B_{\vec{m}}^{+}(0)\right\rangle\right\rangle
\end{aligned}
$$

It should be noticed out that the higher order Green's functions of the type $\left\langle\left\langle B^{+} B B \mid B^{+} B^{+} B\right\rangle\right\rangle$ are rejected, since they are proportional to the square of Bozon concentration $\left\langle B^{+} B\right\rangle$. It means that in further analysis of the Green's function all contributions proportional to the square of Bozon concentration will be rejected. Besides, the concentrations of elementary excitations in ideal structure are not dependent on the lattice point index. ${ }^{1,2}$ So, formula (19) can be written in the following way:

$$
\begin{aligned}
& \left\langle\left\langle P_{\vec{n}}(t) \mid P_{\vec{m}}^{+}(0)\right\rangle\right\rangle \\
& \quad \approx\left(1-4\left\langle B^{+} B\right\rangle^{(0)}\right)\left\langle\left\langle B_{\vec{n}}(t) \mid B_{\vec{m}}^{+}(0)\right\rangle\right\rangle
\end{aligned}
$$

The Green's functions containing four Bose operators can be decoupled, in accordance with Wick's theorem, as follows:

$$
\begin{aligned}
\left\langle\left\langle B_{\vec{a}}^{+}(t)\right.\right. & \left.B_{\vec{a}}(t) B_{\vec{b}}(t)\left|B_{\vec{c}}^{+}(0)\right\rangle\right\rangle \\
= & \left\langle B_{\vec{a}}^{+} B_{\vec{a}}\right\rangle^{(0)}\left\langle\left\langle B_{\vec{b}}(t) \mid B_{\vec{c}}^{+}(0)\right\rangle\right\rangle \\
& \quad\left\langle B_{\vec{a}}^{+} B_{\vec{b}}\right\rangle^{(0)}\left\langle\left\langle B_{\vec{a}}(t) \mid B_{\vec{c}}^{+}(0)\right\rangle\right\rangle
\end{aligned}
$$

The notations $\left\langle B^{+} B\right\rangle^{(0)}$ means that average value of Bozon occupation number is taken over canonical ensemble with zero order Hamiltonian (11). In this Hamiltonian Pauli operators are substituted with Bose operators and the last term on the right-hand side of (11) is rejected.

In the correlator in (14) will be used the approximation $\left\langle P^{+} P\right\rangle \approx\left\langle B^{+} B\right\rangle^{(0)}$. In this way the Eq. (14) becomes:

$$
\begin{aligned}
& i \hbar \frac{d}{d t} G_{\vec{n}, \vec{m}}(t) \\
& =i \hbar \delta(t) \delta_{\vec{n}, \vec{m}}\left(1+2\left\langle B^{+} B\right\rangle^{0}\right)+3 I G_{\vec{n}, \vec{m}}(t) \\
& \quad-\frac{1}{2} I\left(1-2\left\langle B^{+} B\right\rangle^{(0)}\right)
\end{aligned}
$$

$$
\begin{aligned}
& \times\left(G_{\vec{n}+\vec{a}_{x}, \vec{n}}(t)+G_{\vec{n}-\vec{a}_{x}, \vec{n}}(t)+G_{\vec{n}+\vec{a}_{y}, \vec{n}}(t)+G_{\vec{n}-\vec{a}_{y}, \vec{n}}(t)\right. \\
& \left.\quad+G_{\vec{n}+\vec{a}_{z}, \vec{n}}(t)+G_{\vec{n}-\vec{a}_{z}, \vec{n}}(t)\right) \\
& +I\left(\left\langle B_{\vec{n}}^{+} B_{\vec{n}+\vec{a}_{x}}\right\rangle^{(0)}+\left\langle B_{\vec{n}}^{+} B_{\vec{n}-\vec{a}_{x}}\right\rangle^{(0)}+\left\langle B_{\vec{n}}^{+} B_{\vec{n}+\vec{a}_{y}}\right\rangle^{(0)}\right. \\
& \left.\quad+\left\langle B_{\vec{n}}^{+} B_{\vec{n}-\vec{a}_{y}}\right\rangle^{(0)}+\left\langle B_{\vec{n}}^{+} B_{\vec{n}+\vec{a}_{z}}\right\rangle^{(0)}+\left\langle B_{\vec{n}}^{+} B_{\vec{n}-\vec{a}_{z}}\right\rangle^{(0)}\right) \\
& \times G_{\vec{n}, \vec{m}}(t)-6 I\left\langle B^{+} B\right\rangle^{(0)} G_{\vec{n}, \vec{m}}(t) \\
& -I\left(\left\langle B_{\vec{n}+a_{x}}^{+} B_{\vec{n}}\right\rangle^{(0)} G_{\vec{n}+\vec{a}_{x}, \vec{m}}(t)+\left\langle B_{\vec{n}-a_{x}}^{+} B_{\vec{n}}\right\rangle^{(0)} G_{\vec{n}-\vec{a}_{x}, \vec{m}}(t)\right. \\
& \quad+\left\langle B_{\vec{n}+a_{y}}^{+} B_{\vec{n}}\right\rangle^{(0)} G_{\vec{n}+\vec{a}_{y}, \vec{m}}(t)+\left\langle B_{\vec{n}-a_{y}}^{+} B_{\vec{n}}\right\rangle^{(0)} G_{\vec{n}-\vec{a}_{y}, \vec{m}}(t) \\
& \left.\quad+\left\langle B_{\vec{n}+a_{z}}^{+} B_{\vec{n}}\right\rangle^{(0)} G_{\vec{n}+\vec{a}_{z}, \vec{m}}(t)+\left\langle B_{\vec{n}-a_{z}}^{+} B_{\vec{n}}\right\rangle^{(0)} G_{\vec{n}-\vec{a}_{z}, \vec{m}}(t)\right)
\end{aligned}
$$

In this expression $G$ is Bozon Green's function, i.e.,

$$
G_{\vec{n}, \vec{m}}(t)=\left\langle\left\langle B_{\vec{n}}(t) \mid B_{\vec{m}}^{+}(0)\right\rangle\right\rangle
$$

After Fourier transformations:

$$
\begin{gathered}
G_{\vec{n}, \vec{m}}(t)=\frac{1}{N} \sum_{\vec{k}} e^{i \vec{k}(\vec{n}-\vec{m})} \int_{-\infty}^{+\infty} d \omega e^{-i \omega t} G_{\vec{k}}(\omega) \\
\delta(t)=\int_{-\infty}^{+\infty} d \omega e^{-i \omega t} ; \quad \delta_{\vec{n}, \vec{m}}=\frac{1}{N} \sum_{\vec{k}} e^{-i \vec{k}(\vec{n}-\vec{m})} \\
\left\langle B_{a}^{+} B_{b}\right\rangle^{(0)}=\frac{1}{N} \sum_{\vec{k}}\left\langle B_{\vec{k}}^{+} B_{\vec{k}}\right\rangle e^{-i \vec{k}(\vec{n}-\vec{m})}
\end{gathered}
$$

the Eq. (22) reduces to:

$$
G_{k_{x}, k_{y}, k_{z}}(\omega)=\frac{i}{2 \pi} \frac{1+2\left\langle B^{+} B\right\rangle^{(0)}}{\omega-\omega_{k_{x}, k_{y}, k_{z}}}
$$

where $\omega=E / \hbar$ and $\omega_{k_{x}, k_{y}, k_{z}}=E_{k_{x}, k_{y}, k_{z}} / \hbar$. The energy of the spin waves is given by:

$$
\begin{aligned}
E_{k_{x}, k_{y}, k_{z}}^{(1)}= & E_{k_{x}, k_{y}, k_{z}}^{(0)} \\
& +\frac{2 I}{N} \sum_{q_{x}, q_{y}, q_{z}}\left[\cos a k_{x}+\cos a k_{y}+\cos a k_{z}+\cos a q_{x}\right. \\
& \quad+\cos a q_{y}+\cos a q_{z}-3-\cos a\left(k_{x}-q_{x}\right) \\
& \left.\quad-\cos a\left(k_{y}-q_{y}\right)-\cos a\left(k_{z}-q_{z}\right)\right]
\end{aligned}
$$

where

$$
E_{k_{x}, k_{y}, k_{z}}^{(0)}=2 I\left(\sin ^{2} \frac{a k_{x}}{2}+\sin ^{2} \frac{a k_{y}}{2}+\sin ^{2} \frac{a k_{z}}{2}\right)
$$

The second term in (26) is correction of harmonic energy spin waves, which comes from spin waves interaction (mass operator).

The spectral intensity of Green's function (25) is given by well-known formula:

$$
I_{G}=\frac{G(\omega+i \delta)-G(\omega-i \delta)}{e^{\hbar \omega / \Theta}-1} ; \quad \delta \rightarrow 0^{+} ; \Theta=k_{\mathrm{B}} T
$$


wherefrom it follows:

$$
I_{G}(\omega)=\frac{1+2\left\langle B^{+} B\right\rangle^{(0)}}{e^{\hbar \omega / \Theta}-1} \delta\left(\omega-\omega_{k_{x}, k_{y}, k_{z}}\right)
$$

The correlation function in momentum space is given by:

$$
\begin{aligned}
& \left\langle B_{k_{x}, k_{y}, k_{z}}^{+}(0) B_{k_{x}, k_{y}, k_{z}}(t)\right\rangle \\
& =\int_{-\infty}^{+\infty} d \omega e^{-i \omega t} I_{G}(\omega) \\
& =\frac{1+2\left\langle B^{+} B\right\rangle^{(0)}}{e^{E_{k_{x}, k_{y}, k_{z}}^{(1)} / \Theta}-1} e^{-(i t / \hbar) E_{k_{x}, k_{y}, k_{z}}^{(1)}}
\end{aligned}
$$

where

$$
\left\langle B^{+} B\right\rangle^{(0)}=\frac{1}{N} \sum_{k_{x}, k_{y}, k_{z}} \frac{1}{e^{E_{k_{x}, k_{y}, k_{z}}^{(0)} / \Theta}-1}
$$

The first order Bozon concentration is given, in accordance with (30), by:

$$
\left\langle B_{k_{x}, k_{y}, k_{z}}^{+}(0) B_{k_{x}, k_{y}, k_{z}}(0)\right\rangle=\frac{1+2\left\langle B^{+} B\right\rangle^{(0)}}{e^{E_{k_{x}, k_{y}, k_{z}}^{(1)} / \Theta}-1}
$$

Using formula (18) we can write:

$$
\left\langle P^{+} P\right\rangle=\left\langle B^{+} B\right\rangle^{(1)}-\left\langle B^{+} B^{+} B B\right\rangle
$$

and this, on the basis of Wick's theorem, goes over to:

$$
\left\langle P^{+} P\right\rangle=\left\langle B^{+} B\right\rangle^{(1)}-2\left[\left\langle B^{+} B\right\rangle^{(0)}\right]^{2}
$$

Since (see formula (32))

$$
\left\langle B^{+} B\right\rangle^{(1)}=\frac{1}{N} \sum_{k_{x}, k_{y}, k_{z}} \frac{1+2\left\langle B^{+} B\right\rangle^{(0)}}{e^{E_{k_{x}, k_{y}, k_{z}}^{(1)} / \Theta}-1}
$$

formula (31) reduces to:

$$
\left\langle P^{+} P\right\rangle=\frac{1}{N} \sum_{k_{x}, k_{y}, k_{z}} \frac{1}{e^{E_{k_{x}, k_{y}, k_{z}}^{(1)} / \Theta}-1}
$$

with the rest proportional to $\tau^{11 / 2}$. It means that ordering parameter is given by:

$$
\sigma=1-\frac{2}{N} \sum_{k_{x}, k_{y}, k_{z}} \frac{1}{e^{E_{k_{x}, k_{y}, k_{z}}^{(1)} / \Theta}-1}
$$

Substituting in (36) $E_{k_{x}, k_{y}, k_{z}}^{(1)}$ from the formula (36) and expanding cosine functions up to $|\vec{k}|^{6}$ we obtain well-known Dyson's formula for low temperature magnetization (8).

Since Bozon representation (15) are valid for all possible values of Bozon occupation number the problem of non-physical states in the exposed approach does not arise.

\section{MAGNETIZATION OF ULTRATHIN FILM IN BLOCH'S (HARMONIC) APPROXIMATION}

In this part we shall examine behaviour of ordering parameter of ultrathin magnetic film with spin $S=1 / 2$ in Bloch's (harmonic) approximation. In this approximation Pauli operators $P$ are substituted with Bose operators $B$ and the terms corresponding to dynamical interaction of spin waves is rejected. Consequently, Hamiltonian has the following form:

$$
\begin{array}{r}
H=\frac{1}{2} \sum_{\vec{n}}\left(I_{\vec{n}, \vec{n}+\vec{a}_{x}}+I_{\vec{n}, \vec{n}-\vec{a}_{x}}+I_{\vec{n}, \vec{n}+\vec{a}_{y}}+I_{\vec{n}, \vec{n}-\vec{a}_{y}}\right. \\
\left.+I_{\vec{n}, \vec{n}+\vec{a}_{z}}+I_{\vec{n}, \vec{n}-\vec{a}_{z}}\right) B_{\vec{n}}^{+} B_{\vec{n}} \\
-\frac{1}{2} \sum_{\vec{n}} B_{\vec{n}}^{+}\left(I_{\vec{n}, \vec{n}+\vec{a}_{x}} B_{\vec{n}+\vec{a}_{x}}+I_{\vec{n}, \vec{n}-\vec{a}_{x}} B_{\vec{n}-\vec{a}_{x}}\right. \\
+I_{\vec{n}, \vec{n}+\vec{a}_{y}} B_{\vec{n}+\vec{a}_{y}}+I_{\vec{n}, \vec{n}-\vec{a}_{y}} B_{\vec{n}-\vec{a}_{y}} \\
\left.+I_{\vec{n}, \vec{n}+\vec{a}_{z}} B_{\vec{n}+\vec{a}_{z}}+I_{\vec{n}, \vec{n}-\vec{a}_{z}} B_{\vec{n}-\vec{a}_{z}}\right)
\end{array}
$$

We assume that this film is cut off from simple cubic lattice. It is also assumed that the translational symmetry is disturbed in $z$-direction. In $x, y$ planes this symmetry is conserved. The simplest boundary conditions, consisting in absence of layers $n_{z}=-1$ and $n_{z}=N_{z}+1$ will be taken. It means that the boundary conditions are fomulated as follows:

$$
I_{n_{x}, n_{y}, 0 ; n_{x}, n_{y},-1}=I_{n_{x}, n_{y}, N_{z} ; n_{x}, n_{y}, N_{z}+1}=0
$$

Described magnetic film will be analysed with help of Green's function

$$
g_{\vec{n}, \vec{m}}(t)=\theta(t)\left\langle\left[B_{\vec{n}}(t), B_{\vec{m}}^{+}(0)\right]\right\rangle=\left\langle\left\langle B_{\vec{n}}(t) \mid B_{\vec{m}}^{+}(0)\right\rangle\right\rangle
$$

It should be noticed that calculation technique of Green's functions depending on two indices separately is developed in. ${ }^{31-35}$

Differentiating (39) with respect to time and using equations of motion for operator $B_{\vec{n}}(t)$, we obtain the following equation

$$
\begin{aligned}
& i \hbar \frac{d}{d t} g_{n_{x}, n_{y}, n_{z} ; m_{x}, m_{y}, m_{z}}(t) \\
& =i \hbar \delta(t) \delta_{n_{x}, n_{y}, n_{z} ; m_{x}, m_{y}, m_{z}}+3 \operatorname{Ig}_{n_{x}, n_{y}, n_{z} ; m_{x}, m_{y}, m_{z}}(t) \\
& \quad-\frac{1}{2} I\left[g_{n_{x}+1, n_{y}, n_{z} ; m_{x}, m_{y}, m_{z}}(t)+g_{n_{x}-1, n_{y}, n_{z} ; m_{x}, m_{y}, m_{z}}(t)\right. \\
& \quad+g_{n_{x}, n_{y}+1, n_{z} ; m_{x}, m_{y}, m_{z}}(t)+g_{n_{x}, n_{y}-1, n_{z} ; m_{x}, m_{y}, m_{z}}(t) \\
& \left.\quad+g_{n_{x}, n_{y}, n_{z}+1 ; m_{x}, m_{y}, m_{z}}(t)+g_{n_{x}, n_{y}, n_{z}-1 ; m_{x}, m_{y}, m_{z}}(t)\right]
\end{aligned}
$$

where

$$
I=I_{n_{x} \pm 1, n_{y}, n_{z}}=I_{n_{x}, n_{y} \pm 1, n_{z}}=I_{n_{x}, n_{y}, n_{z} \pm 1}
$$


Here will be carried out transition to frequency space by means of Fourier transformations

$$
\begin{aligned}
g_{n_{x}, n_{y}, n_{z} ; m_{x}, m_{y}, m_{z}}(t) & =\int_{-\infty}^{+\infty} d \omega e^{-i \omega t} g_{n_{x}, n_{y}, n_{z} ; m_{x}, m_{y}, m_{z}}(\omega) \\
\delta(t) & =1 / 2 \pi \int_{-\infty}^{+\infty} d \omega e^{-i \omega t}
\end{aligned}
$$

After these transformations (40) becomes:

$$
\begin{aligned}
& (E-3 I) g_{n_{x}, n_{y}, n_{z} ; m_{x}, m_{y}, m_{z}}(\omega) \\
& =\frac{i \hbar}{2 \pi} \delta(t) \delta_{n_{x}, m_{x}} \delta_{n_{y}, m_{y}} \delta_{n_{z}, m_{z}} \\
& \quad-\frac{1}{2} I\left[g_{n_{x}+1, n_{y}, n_{z} ; m_{x}, m_{y}, m_{z}}(t)+g_{n_{x}-1, n_{y}, n_{z} ; m_{x}, m_{y}, m_{z}}(t)\right. \\
& \quad+g_{n_{x}, n_{y}+1, n_{z} ; m_{x}, m_{y}, m_{z}}(t)+g_{n_{x}, n_{y}-1, n_{z} ; m_{x}, m_{y}, m_{z}}(t) \\
& \left.\quad+g_{n_{x}, n_{y}, n_{z}+1 ; m_{x}, m_{y}, m_{z}}(t)+g_{n_{x}, n_{y}, n_{z}-1 ; m_{x}, m_{y}, m_{z}}(t)\right]
\end{aligned}
$$

Taking into account that considered film is translationally invariant in $x, y$ planes, we introduce the transformations:

$$
\begin{gathered}
g_{n_{x}, n_{y}, n_{z} ; m_{x}, m_{y}, m_{z}}(\omega)=\frac{1}{N_{x} N_{y}} \sum_{k_{x}, k_{y}} \gamma_{n_{z}, m_{z}} e^{i a k_{x}\left(n_{x}-m_{x}\right)+i a k_{y}\left(n_{y}-m_{y}\right)} \\
\delta_{n_{x}, m_{x}}=\frac{1}{N_{x}} \sum_{k_{x}} e^{i a k_{x}\left(n_{x}-m_{x}\right)} \\
\delta_{n_{y}, m_{y}}=\frac{1}{N_{y}} \sum_{k_{y}} e^{i a k_{y}\left(n_{y}-m_{y}\right)}
\end{gathered}
$$

After these transformations relation (43) becomes:

$$
\begin{aligned}
& \frac{1}{2} I\left[\gamma_{n_{z}+1, m_{z}}\left(k_{x}, k_{y}, \omega\right)+\gamma_{n_{z}-1, m_{z}}\left(k_{x}, k_{y}, \omega\right)\right] \\
& +\left[E-3 I+I\left(\cos a k_{x}+\cos a k_{y}\right)\right] \gamma_{n_{z}, m_{z}}\left(k_{x}, k_{y}, \omega\right) \\
& \quad=\frac{i \hbar}{2 \pi} \delta_{n_{z}, m_{z}}
\end{aligned}
$$

This equation is valid for an ideal structure. Since we deal with thin film the boundary conditions (41) have to be included. The application of boundary conditions translates (45) into system of three difference equations

$$
\begin{gathered}
\frac{1}{2} I\left(\gamma_{n_{z}+1, m_{z}}+\gamma_{n_{z}-1, m_{z}}\right)+\rho \gamma_{n_{z}, m_{z}}=\frac{i \hbar}{2 \pi} \delta_{n_{z}, m_{z}} ; \\
1 \leq n_{z} \leq N_{z}-1 \\
\frac{1}{2} I \gamma_{1, m_{z}}+\left(\rho+\frac{1}{2} I\right) \gamma_{0, m_{z}}=\frac{i \hbar}{2 \pi} \delta_{0, m_{z}} ; \quad n_{z}=0 \\
\frac{1}{2} I \gamma_{N_{z}-1, m_{z}}+\left(\rho+\frac{1}{2} I\right) \gamma_{N_{z}, m_{z}}=\frac{i \hbar}{2 \pi} \delta_{N_{z}, m_{z}} ; \quad n_{z}=N_{z}
\end{gathered}
$$

where

$$
\rho=E-3 I+I\left(\cos a k_{x}+\cos a k_{y}\right)
$$

It can be easily concluded that the transformation

$$
\gamma_{n_{z}, m_{z}}\left(k_{x}, k_{y}, \omega\right)=\sum_{\lambda=1}^{N_{z}} c_{m_{z}, \lambda} f_{n_{z}, \lambda}
$$

where

$$
f_{n_{z}, \lambda}=\sin \left(n_{z}+1\right) \xi_{\lambda}-\sin n_{z} \xi_{\lambda}
$$

and

$$
\xi_{\lambda}=\frac{\pi \lambda}{N_{z}+1} ; \quad \lambda=1,2,3, \ldots, N_{z}
$$

reduces all Eqs. (46)-(48) into the unique form:

$$
\begin{gathered}
\sum_{\lambda=1}^{N_{z}}\left(I \cos \xi_{\lambda}+\rho\right) C_{\lambda, m_{z}}\left(k_{x}, k_{y}, \omega\right) f_{n_{z}, \lambda}=\frac{i \hbar}{2 \pi} \delta_{n_{z}, m_{z}} \\
n_{z}=0,1,2, \ldots, N_{z}
\end{gathered}
$$

It should be pointed out that $\lambda$ may not take values 0 and $N_{z}+1$ since it leads to $\gamma \equiv 0$.

The Kronecker symbol $\delta_{n_{z}, m_{z}}$ will be taken in the following form:

$$
\delta_{n_{z}, m_{z}}=\sum_{\lambda=1}^{N_{z}} f_{n_{z}, \lambda} r_{m_{z}, \lambda}
$$

Functions $r_{m_{z}, \lambda}$ are the solutions of the system of algebraic equations obtained from the conditions $\delta_{n_{z}, n_{z}}=1$ and $\delta n_{z}, m_{z}=0$.

Taking

$$
c_{m_{z}, \lambda}\left(k_{x}, k_{y}, \omega\right)=q_{\lambda}\left(k_{x}, k_{y}, \omega\right) r_{m_{z}, \lambda}
$$

and combining (53), (54) into (55) we find that

$$
q_{\lambda}\left(k_{x}, k_{y}, \omega\right)=\frac{i}{2 \pi} \frac{1}{\omega-\omega_{k_{x}, k_{y}, \lambda}^{(0)}}
$$

where

$$
\begin{gathered}
\omega=\frac{E}{\hbar} ; \quad \omega_{k_{x}, k_{y}, \lambda}^{(0)}=\frac{E_{k_{x}, k_{y}, \lambda}^{(0)}}{\hbar} \\
E_{k_{x}, k_{y}, \lambda}^{(0)}=2 I\left(\sin ^{2} \frac{a k_{x}}{2}+\sin ^{2} \frac{a k_{y}}{2}+\sin ^{2} \frac{\xi_{\lambda}}{2}\right)
\end{gathered}
$$

Substituting (56) into (55) and including obtained result into (50), we have:

$$
\gamma_{n_{z}, m_{z}}\left(k_{x}, k_{y}, \omega\right)=\frac{i}{2 \pi} \sum_{\lambda=1}^{N_{z}} \frac{\Omega_{\lambda}\left(n_{z}, m_{z}\right)}{\omega-\omega_{k_{x}, k_{y}, \lambda}^{(0)}}
$$

where

$$
\Omega_{\lambda}\left(n_{z}, m_{z}\right)=f_{n_{z}, \lambda} r_{m_{z}, \lambda}
$$


The inclusion of (58) into (44) leads to the final expression of Green's function $g$ :

$$
\begin{aligned}
g_{n_{x}, n_{y}, n_{z} ; m_{x}, m_{y}, m_{z}}(\omega)= & \frac{i}{2 \pi} \frac{1}{N_{x} N_{y}} \cdot \sum_{k_{x}, k_{y}} e^{i a k_{x}\left(n_{x}-m_{x}\right)+i a k_{y}\left(n_{y}-m_{y}\right)} \\
& \times \sum_{\lambda=1}^{N_{z}} \frac{\Omega_{\lambda}\left(n_{z}, m_{z}\right)}{\omega-\omega_{k_{x}, k_{y}, \lambda}^{(0)}}
\end{aligned}
$$

Spectral intensity of this Green's function is given by the general formula (29), wherefrom it follows:

$$
\begin{aligned}
I_{g}(\omega)= & \frac{1}{N_{x} N_{y}} \sum_{k_{x}, k_{y}} \sum_{\lambda=1}^{2} e^{i a k_{x}\left(n_{x}-m_{x}\right)+i a k_{y}\left(n_{y}-m_{y}\right)} \\
& \times \sum_{\lambda=1}^{N_{z}} \Omega_{\lambda}\left(n_{z}, m_{z}\right) \frac{\delta\left(\omega-\omega_{k_{x}, k_{y}, \lambda}^{(0)}\right)}{e^{\hbar \omega / \Theta}-1}
\end{aligned}
$$

The correlation function is given by:

$$
\left\langle B_{m_{x}, m_{y}, m_{z}}^{+}(0) B_{n_{x}, n_{y}, n_{z}}(t)\right\rangle^{(0)}=\int_{-\infty}^{+\infty} d \omega e^{-i \omega t} I_{g}(\omega)
$$

wherefrom we obtain:

$$
\begin{aligned}
\left\langle B_{m_{x}, m_{y}, m_{z}}^{+}(0) B_{n_{x}, n_{y}, n_{z}}(t)\right\rangle^{(0)} & \\
= & \frac{1}{N_{x} N_{y}} \sum_{k_{x}, k_{y}} \sum_{\lambda=1}^{N_{z}} \frac{\Omega_{\lambda}\left(n_{z}, m_{z}\right)}{e^{E_{k_{x}, k_{y}, \lambda}^{(0)} / \Theta}-1} \\
& \times e^{i a k_{x}\left(n_{x}-m_{x}\right)+i a k_{y}\left(n_{y}-m_{y}\right)-i t \omega_{k_{x}, k_{y}, \lambda}^{(0)}}
\end{aligned}
$$

This formula for $n_{x}=m_{x}, n_{y}=m_{y}, n_{z}=m_{z}$ and $t=0$ gives the expression for Bozon concentration:

$$
\begin{aligned}
& \left\langle B_{n_{x}, n_{y}, n_{z}}^{+}(0) B_{n_{x}, n_{y}, n_{z}}(0)\right\rangle^{(0)} \\
& \quad=\frac{1}{N_{x} N_{y}} \sum_{k_{x}, k_{y}} \sum_{\lambda=1}^{N_{z}} \frac{\Omega_{\lambda}\left(n_{z}, n_{z}\right)}{e^{E_{k_{x}, k_{y}, \lambda}^{(0)} / \Theta}-1}
\end{aligned}
$$

At this stage of analysis it is necessary to point out that the transition from configurational space $n_{z}$ to momentum space $(\lambda)$ is not the isomorphic one. The momentum space is narrower than configurational one since for $n_{z}=$ $0,1,2,3, \ldots, N_{z}$. the momentum index $\lambda$ takes the following set of values $\lambda=1,2,3, \ldots, N_{z}$. This is the most important property of nanostructures, which will be discussed in more detail in further.

The mentioned non-isomorphism leads to autoreduction of the film since the spin waves do not propagate in one of its layers. In the "empty" layer appear some localized spin excitations, probably.

For clearness we shall consider three-layer film where the layers in $z$-direction are denoted with $n_{z}=0,1,2$. Since momentum index $\lambda$ takes the values $\lambda=1,2$, the spin waves propagate in one of three possible two layer subfilms: $n_{z}=0, n_{z}=1 ; n_{z}=1, n_{z}=2$ and $n_{z}=0, n_{z}=2$.
In further for subfilms will be used notations $(0,1),(1,2)$ and $(0,2)$, respectively.

For subfilm $(0,1)$ in accordance with $(63)$ we have:

$$
\left\langle\underset{n_{z}=0}{B^{+} B}\right\rangle^{(0)}=\sum_{\lambda=1}^{2} \Omega_{\lambda}^{01}(0,0) F_{\lambda}
$$

where

$$
\Omega_{1}^{01}(0,0)=f_{01} r_{01}=1 ; \quad \Omega_{2}^{01}(0,0)=f_{02} r_{02}=0
$$

and

$$
\begin{gathered}
F_{\lambda}=\frac{1}{N_{x} N_{y}} \sum_{k_{x}, k_{y}} \frac{1}{e^{E_{k_{x}, k_{y}, \lambda}^{(0)} / \Theta}-1}=\tau Z_{1}\left(\frac{2 \lambda-1}{4 \pi \tau}\right) \\
\tau=\frac{\Theta}{2 \pi I} ; \quad Z_{p}(q)=\sum_{n=1}^{\infty} \frac{e^{-n q}}{n^{p}}
\end{gathered}
$$

For $n_{z}=1$ we have:

$$
\left\langle\underset{n_{z}=1}{B^{+} B}\right\rangle^{(0)}=\sum_{\lambda=1}^{2} \Omega_{\lambda}^{01}(1,1) F_{\lambda}, t
$$

where

$$
\Omega_{1}^{01}(1,1)=f_{11} r_{11}=0 ; \quad \Omega_{2}^{01}(1,1)=f_{12} r_{12}=1
$$

For subfilm $(1,2)$ the Bozon concentrations are given by:

$$
\left\langle\underset{n_{z}=1}{B^{+} B}\right\rangle^{(0)}=\sum_{\lambda=1}^{2} \Omega_{\lambda}^{12}(1,1) F_{\lambda}
$$

where

$$
\Omega_{1}^{12}(1,1)=f_{11} r_{11}=0 ; \quad \Omega_{2}^{12}(1,1)=f_{12} r_{12}=1
$$

and

$$
\left\langle\underset{n_{z}=2}{B^{+} B}\right\rangle^{(0)}=\sum_{\lambda=1}^{2} \Omega_{\lambda}^{12}(2,2) F_{\lambda}
$$

where

$$
\Omega_{1}^{12}(2,2)=f_{21} r_{21}=1 ; \quad \Omega_{2}^{12}(2,2)=f_{22} r_{22}=0
$$

Finally, for subfilm $(0,2)$ Bozon concentrations are given by:

$$
\left\langle\underset{n_{z}=0}{\left\langle B^{+} B\right.}\right\rangle^{(0)}=\sum_{\lambda=1}^{2} \Omega_{\lambda}^{02}(0,0) F_{\lambda}
$$

where

$$
\Omega_{1}^{02}(0,0)=f_{01} r_{01}=\frac{1}{2} ; \quad \Omega_{2}^{02}(0,0)=f_{02} r_{02}=\frac{1}{2}
$$

and

$$
\left\langle\underset{n_{z}=2}{\left.B^{+} B\right\rangle^{(0)}}=\sum_{\lambda=1}^{2} \Omega_{\lambda}^{02}(2,2) F_{\lambda}\right.
$$

where

$$
\Omega_{1}^{02}(2,2)=f_{21} r_{21}=\frac{1}{2} ; \quad \Omega_{2}^{02}(2,2)=f_{22} r_{22}=\frac{1}{2}
$$


It is useful for further analyses to quote Bose concentrations in subfilms as well as some of correlation functions. From the formulas (64)-(76) we obtain the following expressions for Bozon concentrations:

$$
\begin{gathered}
\left\langle\underset{n_{z}=0}{\left\langle B^{+} B\right.}\right\rangle_{01}^{(0)}=\underset{\substack{n_{z}=2 \\
B_{12}^{+} B}}{\langle(0)}=\tau Z_{1}\left(\frac{1}{4 \pi \tau}\right) \\
\left.\left\langle\underset{n_{z}=1}{\left\langle B^{+} B\right.}\right\rangle_{01}^{(0)}=\underset{n_{z}=1}{\left\langle B^{+} B\right.}\right\rangle_{12}^{(0)}=\tau Z_{1}\left(\frac{3}{4 \pi \tau}\right) \\
\underset{n_{z}=0}{\left\langle B_{02}^{+} B\right\rangle_{02}^{(0)}}=\underset{\substack{n_{z}=2 \\
\left\langle B^{+} B\right.}}{(0)}=\frac{1}{2} \tau\left[Z_{1}\left(\frac{1}{4 \pi \tau}\right)+Z_{1}\left(\frac{3}{4 \pi \tau}\right)\right]
\end{gathered}
$$

The transitions $(0,1)$ are characterized by correlation functions $\left\langle B_{n_{x}, n_{y}, 1}^{+} B_{n_{x}, n_{y}, 0}\right\rangle$ and $\left\langle B_{n_{x}, n_{y}, 0}^{+} B_{n_{x}, n_{y}, 1}\right\rangle$. The values of these correlation functions are the following:

$$
\begin{array}{r}
\left\langle B_{n_{x}, n_{y}, 1}^{+} B_{n_{x}, n_{y}, 0}\right\rangle=\left\langle B_{n_{x}, n_{y}, 1}^{+} B_{n_{x}, n_{y}, 2}\right\rangle \\
=\tau\left[Z_{1}\left(\frac{1}{4 \pi \tau}\right)-Z_{1}\left(\frac{3}{4 \pi \tau}\right)\right] \\
\left\langle B_{n_{x}, n_{y}, 0}^{+} B_{n_{x}, n_{y}, 1}\right\rangle=\left\langle B_{n_{x}, n_{y}, 2}^{+} B_{n_{x}, n_{y}, 1}\right\rangle=0
\end{array}
$$

It is interesting to note that the transitions of excitations from boundary layers 0 and 2 to the central layer 1 are different from zero, while the transitions from central layer to boundary layers are "forbidden," i.e., equal to zero. Since the concentrations of excitations in boundary layers are higher than the concentration in central layer, the results (78) mean that in the film exists tending to energy equilibrium since the spin wave energy "flows" from higher energy places to the places of lower energy. This result is physically expected.

The results (77) for Bozon concentrations are in full correspondence with film geometry. This essentially simplifies calculations. Knowing concentrations and connected with them characteristics of one of subfilms, say $(0,1)$, we immediately can find the same for other subfilms.

\section{THE INFLUENCE OF MAGNON-MAGNON INTERACTION IN THIN FILMS TO BLOCH'S MAGNETIZATION}

We start from the Hamiltonian (11) and it will be analysed by means of paulion Green's function (12). Boundary conditions, consisting in absence of layers $n_{z}=-1$ and $n_{z}=N_{z}+1$ will be assumed. It means that they are given by the formula (38). Notations (41) will be used, also.

Differentiating paulion Green's function with respect to time and using the equation of motion $\dot{P}_{\vec{n}}=$ $(1 /(i \hbar))\left[P_{\vec{n}}, H\right]$ we obtain the Eq. (14).

Further analysis will be carried out using exact Bose representation of Pauli operators (15). In low temperature range, as it was shown in the Section 2 it is sufficient to use approximate expressions (16)-(18) instead of (15).
The use of mentioned approximate formulas is described in Section 2 as well as the decoupling procedure of higher order Bozon Green's functions and therefore we shall not repeat it here. The equation for Fourier-transform $G_{\vec{n}, \vec{m}}(\omega)$ the Bozon Green's function:

$$
G_{\vec{n}, \vec{m}}(t)=\left\langle\left\langle B_{\vec{n}}(t) \mid B_{\vec{m}}^{+}(0)\right\rangle\right\rangle
$$

obtained in above described way is the following:

$$
\begin{aligned}
& E G_{\vec{n}, \vec{m}}(\omega) \\
& =\frac{i \hbar}{2 \pi}\left(1+2\left\langle B^{+} B\right\rangle^{(0)}\right) \delta_{\vec{n}, \vec{m}}+3 I G_{\vec{n}, \vec{m}}(\omega)-\frac{I}{2}\left(1-2\left\langle B^{+} B\right\rangle^{(0)}\right) \\
& \times\left[\left(G_{\vec{n}+\vec{a}_{x}, \vec{m}}(\omega)+G_{\vec{n}-\vec{a}_{x}, \vec{m}}(\omega)+G_{\vec{n}+\vec{a}_{y}, \vec{m}}(\omega)\right.\right. \\
& \left.+G_{\vec{n}-\vec{a}_{y}, \vec{m}}(\omega)+G_{\vec{n}+\vec{a}_{z}, \vec{m}}(\omega)+G_{\vec{n}-\vec{a}_{z}, \vec{m}}(\omega)\right]+I G_{\vec{n}, \vec{m}}(\omega) \\
& \times\left(\left\langle B_{\vec{n}}^{+} B_{\vec{n}+\vec{a}_{x}}\right\rangle^{(0)}+\left\langle B_{\vec{n}}^{+} B_{\vec{n}-\vec{a}_{x}}\right\rangle^{(0)}+\left\langle B_{\vec{n}}^{+} B_{\vec{n}+\vec{a}_{y}}\right\rangle^{(0)}\right. \\
& +\left\langle B_{\vec{n}}^{+} B_{\vec{n}-\vec{a}_{y}}\right\rangle^{(0)}+\left\langle B_{\vec{n}}^{+} B_{\vec{n}+\vec{a}_{z}}\right\rangle^{(0)}+\left\langle B_{\vec{n}}^{+} B_{\vec{n}-\vec{a}_{z}}\right\rangle^{(0)} \\
& -\left\langle B_{\vec{n}+\vec{a}_{x}}^{+} B_{\vec{n}+\vec{a}_{x}}\right\rangle^{(0)}-\left\langle B_{\vec{n}-\vec{a}_{x}}^{+} B_{\vec{n}-\vec{a}_{x}}\right\rangle^{(0)}-\left\langle B_{\vec{n}+\vec{a}_{y}}^{+} B_{\vec{n}+\vec{a}_{y}}\right\rangle^{(0)} \\
& \left.-\left\langle B_{\vec{n}-\vec{a}_{y}}^{+} B_{\vec{n}-\vec{a}_{y}}\right\rangle^{(0)}-\left\langle B_{\vec{n}+\vec{a}_{z}}^{+} B_{\vec{n}+\vec{a}_{z}}\right\rangle^{(0)}-\left\langle B_{\vec{n}-\vec{a}_{z}}^{+} B_{\vec{n}-\vec{a}_{z}}\right\rangle^{(0)}\right) \\
& -I\left(\left\langle B_{\vec{n}+\vec{a}_{x}}^{+} B_{\vec{n}}\right\rangle^{(0)} G_{\vec{n}+\vec{a}_{x}, \vec{m}}(\omega)+\left\langle B_{\vec{n}-\vec{a}_{x}}^{+} B_{\vec{n}}\right\rangle^{(0)} G_{\vec{n}-\vec{a}_{x}, \vec{m}}(\omega)\right. \\
& +\left\langle B_{\vec{n}+\vec{a}_{y}}^{+} B_{\vec{n}}\right\rangle^{(0)} G_{\vec{n}+\vec{a}_{y}, \vec{m}}(\omega)+\left\langle B_{\vec{n}-\vec{a}_{y}}^{+} B_{\vec{n}}\right\rangle^{(0)} G_{\vec{n}-\vec{a}_{y}, \vec{m}}(\omega) \\
& \left.+\left\langle B_{\vec{n}+\vec{a}_{z}}^{+} B_{\vec{n}}\right\rangle^{(0)} G_{\vec{n}+\vec{a}_{z}, \vec{m}}(\omega)+\left\langle B_{\vec{n}-\vec{a}_{z}}^{+} B_{\vec{n}}\right\rangle^{(0)} G_{\vec{n}-\vec{a}_{z}, \vec{m}}(\omega)\right)
\end{aligned}
$$

It should be noticed that in the correlator $(i \hbar / 2 \pi)$. $\left(1+2\left\langle B_{\vec{n}}^{+} B_{\vec{n}}\right\rangle\right) \delta_{\vec{n}, \vec{m}}$ the configurational index of Bozon concentration $\left\langle B^{+} B\right\rangle^{(0)}$ is not specified. The reason for this is the fact that the correlator serves for estimate of the error in the transition

$$
\left\langle P^{+} P\right\rangle=\left\langle B^{+} B\right\rangle^{(1)}-2\left[\left\langle B^{+} B\right\rangle^{(0)}\right]^{2}
$$

which was done in the section two. The specification of configurational index does not influence to the mentioned estimate, what will be shown later. On the other hand, the zero order Bozon concentrations as well as correlation functions figuring in the rest of the right-hand side of (79) will be determined by means of the formulae (77) and (78). For Bozon concentrations will be taken arithmetic averages over corresponding subfilms, while for correlation functions the formulae (78) will be applied directly. The translatory invariance in $x, y$ plane will be included in calculations by means of formulae:

$$
\begin{gathered}
G_{n_{x}, n_{y}, n_{z} ; m_{x}, m_{y}, m_{z}}(\omega) \\
=\frac{1}{N_{x} N_{y}} \sum_{k_{x}, k_{y}} \alpha_{n_{z}, m_{z}}\left(k_{x}, k_{y}, \omega\right) e^{i a k_{x}\left(n_{x}-m_{x}\right)+i a k_{y}\left(n_{y}-m_{y}\right)} \\
\delta_{n_{x}, m_{x}}=\frac{1}{N_{x}} \sum_{k_{x}} e^{i a k_{x}\left(n_{x}-m_{x}\right)} \\
\delta_{n_{y}, m_{y}}=\frac{1}{N_{y}} \sum_{k_{y}} e^{i a k_{y}\left(n_{y}-m_{y}\right)}
\end{gathered}
$$




\section{Sajfert et al.}

The further analysis will be related to three-layer film, only. The reason for this lies in the fact that the calculations are very tedious even in the case of three-layer film. The calculations for multi-layer films catastrophically rapidly increase with the increase of number of layers. On the other hand the calculation procedure exposed, allows the formulation of fundamental set of equations for film with arbitrary number of layers without any problem.

For three-layer film and boundary conditions (25) we obtain the following system of difference equations

$$
\begin{array}{r}
n_{z}=1 \quad J\left(\alpha_{2, m_{z}}+\alpha_{0, m_{z}}\right)+\rho \alpha_{1, m_{z}} \\
=\frac{i \hbar}{2 \pi}\left(1+2\left\langle B^{+} B\right\rangle^{(0)}\right) \delta_{1, m_{z}} \\
n_{z}=0 \quad(J-\Delta J) \alpha_{1, m_{z}}+(\rho+\Delta \rho) \alpha_{0, m_{z}} \\
=\frac{i \hbar}{2 \pi}\left(1+2\left\langle B^{+} B\right\rangle^{(0)}\right) \delta_{0, m_{z}} \\
n_{z}=2 \quad(J-\Delta J) \alpha_{1, m_{z}}+(\rho+\Delta \rho) \alpha_{2, m_{z}} \\
=\frac{i \hbar}{2 \pi}\left(1+2\left\langle B^{+} B\right\rangle^{(0)}\right) \delta_{2, m_{z}}
\end{array}
$$

The notations in upper equations are the following:

$$
\begin{gathered}
\rho=E-2 I\left(\sin ^{2} \frac{a k_{x}}{2}+\sin ^{2} \frac{a k_{y}}{2}\right) \\
-I+\frac{1}{2} I\left(F_{1}+3 F_{2}\right)-2 I \Phi_{2} \\
\Delta \rho=\frac{1}{2} I-\frac{1}{2} I\left(F_{1}+F_{2}\right)-\frac{3}{2} I \Phi_{2} \\
\Delta \Phi=\Phi_{1}-\Phi_{2} \\
J=\frac{1}{2} I\left(1-2 F_{2}\right) \\
\Delta J=\frac{1}{4} I \Delta F \\
\Delta F=F_{1}-F_{2}
\end{gathered}
$$

The functions $F$ and $\Phi$ are given by:

$$
\begin{aligned}
F_{\lambda}= & \frac{1}{N_{x} N_{y}} \sum_{k_{x}, k_{y}} \frac{1}{e^{E_{k_{x}, k_{y}, \lambda}^{(0)}}-1} \\
= & \tau Z_{1}\left(\frac{2 \lambda-1}{4 \pi \tau}\right)+\frac{\pi}{2} \tau^{2} Z_{2}\left(\frac{2 \lambda-1}{4 \pi \tau}\right)+O\left(\tau^{3} Z\right) ; \\
& \frac{2 \lambda-1}{2} \frac{I}{\Theta}=\frac{2 \lambda-1}{4 \pi \tau}
\end{aligned}
$$

and

$$
\begin{aligned}
\Phi_{\lambda} & =\frac{1}{N_{x} N_{y}} \sum_{q_{x}, q_{y}} \frac{M\left(k_{x}, k_{y}, q_{x}, q_{y}\right)}{e^{E_{q_{x}, q_{y}, \lambda}^{(0)}}-1} \\
& =-a k\left[\pi \tau^{3 / 2} Z_{3 / 2}\left(\frac{2 \lambda-1}{4 \pi \tau}\right)-\frac{27}{16} \pi^{2} \tau^{5 / 2} Z_{5 / 2}\left(\frac{2 \lambda-1}{4 \pi \tau}\right)\right.
\end{aligned}
$$

Behaviour of Thin Magnetic Films at Low Temperatures

$$
\begin{gathered}
-(a k)^{2}\left[\frac{3}{4} \pi \tau^{2} Z_{2}\left(\frac{2 \lambda-1}{4 \pi \tau}\right)-\frac{19}{16} \pi^{2} \tau^{3} Z_{3}\left(\frac{2 \lambda-1}{4 \pi \tau}\right)\right] \\
+(a k)^{3}\left[\frac{1}{8} \pi \tau^{3 / 2} Z_{3 / 2}\left(\frac{2 \lambda-1}{4 \pi \tau}\right)\right. \\
\left.-\frac{95}{768} \pi^{2} \tau^{5 / 2} Z_{5 / 2}\left(\frac{2 \lambda-1}{4 \pi \tau}\right)\right] \\
+O\left(\tau^{7 / 2} Z\right)
\end{gathered}
$$

The results (90) and (91) are obtained for

$$
\begin{array}{r}
E_{k_{x}, k_{y}, \lambda}^{(0)}=2 I\left(\sin ^{2} \frac{a k_{x}}{2}+\sin ^{2} \frac{a k_{y}}{2}+\sin ^{2} \frac{\lambda \pi}{6}\right) ; \\
\lambda=1,2
\end{array}
$$

and

$$
\begin{aligned}
M\left(k_{x}, k_{y}, q_{x}, q_{y}\right)= & \cos a k_{x}+\cos a k_{y}+\cos a q_{x} \\
& +\cos a q_{y}-2-\cos a\left(k_{x}-q_{x}\right) \\
& -\cos a\left(k_{y}-q_{y}\right)
\end{aligned}
$$

It can be easily shown that all Eqs. (81)-(83) reduce to the unique one

$$
\begin{gathered}
\sum_{\lambda=1}^{2}\left(2 J \cos \varphi_{\lambda}+\rho\right) a_{m_{z}, \lambda}\left(k_{x}, k_{y}, \omega\right) S_{n_{z}, \lambda}=\frac{i \hbar}{2 \pi} \delta_{n_{z}, m_{z}} \\
n_{z}, m_{z} \in\{0,1,2\}
\end{gathered}
$$

by the substitution:

$$
\alpha_{n_{z}, m_{z}}=\sum_{\lambda=1}^{2} a_{m_{z}, \lambda} S_{n_{z, \lambda}}
$$

where

$$
S_{n_{z}, \lambda}=\sin \left(n_{z}+1\right) \varphi_{\lambda}+Y_{\lambda} \sin n_{z} \varphi_{\lambda}
$$

and

$$
Y_{\lambda}=-1+\left[\left(1+\cos \varphi_{\lambda}^{(0)}\right) \Delta F+3 \Delta \Phi\right]
$$

if the parameters $\varphi_{\lambda}$ satisfy the following equation:

$$
\begin{gathered}
\frac{1}{2} \sin 3 \varphi_{\lambda}-\sin 3 \varphi_{\lambda} \cos \varphi_{\lambda}+\sin 2 \varphi_{\lambda} \cos \varphi_{\lambda}-\frac{1}{2} \sin \varphi_{\lambda} \\
+\frac{5 \sqrt{3}}{8} \Delta F+\frac{3 \sqrt{3}}{2} \Delta \Phi=0
\end{gathered}
$$

Using the approximate formulae:

$$
\begin{array}{ll}
\sin (x+\varepsilon) \approx \sin x+\varepsilon \cos x ; & \varepsilon \ll x \\
\cos (x+\varepsilon) \approx \cos x-\varepsilon \sin x ; & \varepsilon \ll x
\end{array}
$$

we obtain the following expressions for parameters $\varphi_{\lambda}$ :

$$
\begin{aligned}
& \varphi_{1}=\frac{\pi}{3}+\delta_{1} ; \quad \delta_{1}=\frac{5 \sqrt{3}}{12} \Delta F+\sqrt{3} \Delta \Phi ; \\
& \varphi_{2}=\frac{2 \pi}{3}+\delta_{2} ; \quad \delta_{2}=\frac{\sqrt{3}}{36} \Delta F+\frac{\sqrt{3}}{3} \Delta \Phi
\end{aligned}
$$


The Kronecker symbol in (94) will be taken in the form:

$$
\delta_{n_{z}, m_{z}}=\sum_{\lambda=1}^{2} S_{n_{z}, \lambda} T_{m_{z}, \lambda}
$$

It was earlier said that if we know concentrations in one of subfilms $(0,1),(1,2)$ or $(0,2)$, they are quite sufficient for determining the concentrations in other two subfilms and therefore we shall look for the solutions of subfilm $(0,1)$, only. For this subfilm the Eq. (101) gives the following values for parameters $T$ :

$$
\begin{aligned}
T_{01}^{01}=\frac{S_{12}^{01}}{D} ; \quad T_{02}^{01} & =\frac{S_{11}^{01}}{D} ; \quad T_{11}^{01}=\frac{S_{02}^{01}}{D} ; \quad T_{12}^{01}=\frac{S_{01}^{01}}{D} \\
D & =S_{01}^{012} S_{12}^{01}-S_{02}^{01} S_{11}^{01}
\end{aligned}
$$

Using the formulae (96), (97) and (100) we obtain:

$$
\begin{gathered}
S_{01}^{01}=\frac{\sqrt{3}}{2}\left(1+\frac{5}{6} \Delta F+2 \Delta \Phi\right) \\
S_{02}^{01}=\frac{\sqrt{3}}{2}\left[1-\left(\frac{1}{6} \Delta F+\frac{1}{3} \Delta \Phi\right)\right] \\
S_{11}^{01}=\frac{23 \sqrt{3}}{24} \Delta F+2 \sqrt{3} \Delta \Phi \\
S_{12}^{01}=-\sqrt{3}\left(1+\frac{17}{72} \Delta F+\Delta \Phi\right)
\end{gathered}
$$

Combining (102) and (103) we find:

$$
\begin{gathered}
S_{01}^{01} T_{01}^{01}=S_{12}^{01} T_{12}^{01}=1-\left(\frac{23}{24} \Delta F+2 \Delta \Phi\right) \\
S_{02}^{01} T_{02}^{01}=S_{11}^{01} T_{11}^{01}=\frac{23}{24} \Delta F+2 \Delta \Phi
\end{gathered}
$$

Substituting (101) into (94) and taking

$$
a_{m_{z}, \lambda}\left(k_{x}, k_{y}, \omega\right)=b_{k_{x}, k_{y}, \lambda}(\omega) T_{m_{z}, \lambda}
$$

we reduce (94) to:

$$
b_{k_{x}, k_{y}, \lambda}(\omega)=\frac{i}{2 \pi} \frac{1+2\left\langle B^{+} B\right\rangle^{(0)}}{\omega-\omega_{k_{x}, k_{y}, \lambda}^{(1)}}
$$

where

$$
\begin{gathered}
\omega_{k_{x}, k_{y}, \lambda}=\frac{1}{\hbar} E_{k_{x}, k_{y}, \lambda}^{(1)} \\
E_{k_{x}, k_{y}, \lambda}^{(1)}=2 I\left(\sin ^{2} \frac{a k_{x}}{2}+\sin ^{2} \frac{a k_{y}}{2}+\sin ^{2} \frac{\varphi_{\lambda}}{2}\right) \\
-\frac{1}{2} I F_{1}-\frac{1}{2} I F_{2}\left(3-4 \cos \frac{\lambda \pi}{3}\right)+2 I \Phi_{2}
\end{gathered}
$$

Combining (95), (105), (106) and (80) we obtain Bozon Green's function of considered system:

$$
\begin{aligned}
G_{n_{x}, n_{y}, n_{z} ; m_{x}, m_{y}, m_{z}}(\omega) & \\
= & \frac{i\left(1+2\left\langle B^{+} B\right\rangle^{(0)}\right)}{2 \pi} \frac{1}{N_{x} N_{y}} \\
& \cdot \sum_{k_{x}, k_{y} \lambda=1}^{2} e^{i a k_{x}\left(n_{x}-m_{x}\right)+i a k_{y}\left(n_{y}-m_{y}\right)} \cdot \frac{S_{n_{z}, \lambda} T_{m_{z}, \lambda}}{\omega-\omega_{k_{x}, k_{y}, \lambda}^{(0)}}
\end{aligned}
$$

Having the formula (108), we can find the spectral intensity, correlation function and Bozon concentrations in the first approximation. We shall quote first order Bozon concentration for subfilm $(0,1)$, only:

$$
\begin{aligned}
\left\langle B^{+} B\right\rangle_{01}^{(1)}= & \left(1+2\left\langle B^{+} B\right\rangle^{(0)}\right) \frac{1}{N_{x} N_{y}} \\
& \cdot \sum_{k_{x}, k_{y}} \sum_{\lambda=1}^{2} \frac{S_{n_{z}, \lambda}^{01} T_{n_{z}, \lambda}^{01}}{e^{E_{k_{x}, k_{y}, \lambda}^{(1)}}-1}
\end{aligned}
$$

Now we shall determine ordering parameter and estimate order of magnitude its rest. The expression for $\sigma$ is given by:

$$
\sigma=1-2\left\langle P^{+} P\right\rangle=1-2\left\langle B^{+} B\right\rangle^{(1)}+4\left[\left\langle B^{+} B\right\rangle^{(0)}\right]^{2}
$$

Taking into account (109) we can write:

$$
\begin{aligned}
\sigma= & 1-\frac{2}{N_{x} N_{y}} \sum_{k_{x}, k_{y}} \sum_{\lambda=1}^{2} \frac{S_{n_{z}, \lambda} T_{n_{z}, \lambda}}{e^{E_{k_{x}, k_{y}, \lambda}^{(1)} / \Theta}-1} \\
& -4\left\langle B^{+} B\right\rangle^{(0)} \Delta\left\langle B^{+} B\right\rangle
\end{aligned}
$$

The last term on the right-hand side (111) will be neglected. It means that ordering parameter will be calculated by means of formula (111) including first two righthand side terms, only.

In order to follow exactness of further calculation we shall estimate order of magnitude the neglected term. Since $\left\langle B^{+} B\right\rangle^{(0)}$ is of the order $\tau Z_{1}$ we shall estimate the order of the magnitude $\Delta\left\langle B^{+} B\right\rangle$, only. Using the formula:

$$
\begin{aligned}
E_{k_{x}, k_{y}, \lambda}^{(1)}= & E_{k_{x}, k_{y}, \lambda}^{(0)}+I\left(\frac{\sqrt{3}}{2} \delta_{\lambda}-\frac{1}{2} F_{1}\right. \\
& \left.-\frac{1}{2} F_{2}\left(3-4 \cos \frac{\lambda \pi}{3}\right)+2 \Phi_{2}\right)
\end{aligned}
$$

we can write

$\Delta\left\langle B^{+} B\right\rangle$

$$
\begin{aligned}
& =\frac{1}{N_{x} N_{y}} \sum_{k_{x}, k_{y} \lambda=1} \sum^{2}\left[\frac{S_{n_{z}, \lambda} T_{n_{z}, \lambda}}{e^{E_{k_{x}, k_{y}, \lambda}^{(1)} / \Theta}-1}-\frac{f_{n_{z}, \lambda} r_{n_{z}, \lambda}}{e^{E_{k_{x}, k_{y}, \lambda}^{(0)}}-1}\right] \\
& \approx \frac{1}{N_{x} N_{y}} \sum_{k_{x}, k_{y} \lambda=1} \sum^{2} e^{-n E_{k_{x}, k_{y}, \lambda}^{(0)} / \Theta}\left\{S _ { n _ { z } , \lambda } T _ { n _ { z } , \lambda } \left[1-\frac{n I}{\Theta}\left(\frac{\sqrt{3}}{2} \delta_{\lambda}-\frac{1}{2} F_{1}\right.\right.\right. \\
& \left.\left.\quad-\frac{1}{2} F_{2}\left(3-4 \cos \frac{\lambda \pi}{3}\right)+2 \Phi_{2}\right]-f_{n_{z}, \lambda} r_{n_{z}, \lambda}\right\}
\end{aligned}
$$

Taking leading terms, only, we find from the last formula that

$$
\Delta\left\langle B^{+} B\right\rangle \sim \frac{1}{16 \pi} \tau Z_{1}\left(\frac{1}{4 \pi \tau}\right) Z_{0}\left(\frac{1}{4 \pi \tau}\right)
$$

This leads to the estimate:

$$
-4\left\langle B^{+} B\right\rangle^{(0)} \Delta\left\langle B^{+} B\right\rangle \sim \frac{1}{4 \pi} \tau^{2} Z_{0}\left(\frac{1}{4 \pi \tau}\right) Z_{1}^{2}\left(\frac{1}{4 \pi \tau}\right)
$$




\section{Sajfert et al.}

so that we can write, finally:

$$
\sigma=1-\frac{2}{N_{x} N_{y}} \sum_{k_{x}, k_{y}} \sum_{\lambda=1}^{2} \frac{S_{n_{z}, \lambda} T_{n_{z}, \lambda}}{e^{E_{k_{x}, k_{y}, \lambda}^{(1)} / \Theta}-1}+O\left(\tau^{2} Z^{3}\right)
$$

The temperature functions $F$ and $\Phi$ appearing during calculation of $\sigma$ are given by the formulas (90) and (91). It should be noticed that the results (90) and (91) are obtained in the following small wave vectors approximation:

$$
\begin{aligned}
\sin ^{2} & \frac{a k_{x}}{2}+\sin ^{2} \frac{a k_{y}}{2} \\
& \approx \frac{a^{2} k^{2}}{4}-\frac{a^{4} k^{4}}{48}\left(\cos ^{4} \varphi+\cos ^{4} \varphi\right)+O\left(k^{6}\right)
\end{aligned}
$$

Since the rest in (90) is of the order $\tau^{3} Z$ it must be used

$$
F^{2}=\tau^{2} Z_{1}^{2}+\pi \tau^{3} Z_{1} Z_{2}+O\left(\tau^{4} Z^{2}\right)
$$

Keeping this in mind we found the following expression for ordering parameters. The ordering parameter for the layer $n_{z}=0$ is:

$$
\sigma^{01}(0)=\sigma_{h}^{01}(0)+\delta_{a} \sigma^{01}(0)
$$

where

$$
\begin{aligned}
\sigma_{h}^{01}(0)= & -2 \tau Z_{1}\left(\frac{1}{4 \pi \tau}\right) \\
& -\pi \tau^{2} Z_{2}\left(\frac{1}{4 \pi \tau}\right)+O\left(\tau^{3} Z\right)
\end{aligned}
$$

and

$$
\delta_{a} \sigma^{01}(0)=a_{1} \tau+a_{2} \tau^{2}+a_{3} \tau^{3}+O\left(\tau^{4} Z^{2}\right)
$$

where

$$
\begin{aligned}
a_{1}= & \frac{1}{8 \pi} Z_{0}\left(\frac{1}{4 \pi \tau}\right) Z_{1}\left(\frac{1}{4 \pi \tau}\right)-\frac{9}{8 \pi} Z_{0}\left(\frac{1}{4 \pi \tau}\right) Z_{1}\left(\frac{3}{4 \pi \tau}\right) \\
a_{2}= & \frac{1}{16}\left[Z_{0}\left(\frac{1}{4 \pi \tau}\right) Z_{2}\left(\frac{1}{4 \pi \tau}\right)+Z_{1}^{2}\left(\frac{1}{4 \pi \tau}\right)\right] \\
& -\frac{9}{16}\left[Z_{0}\left(\frac{1}{4 \pi \tau}\right) Z_{2}\left(\frac{3}{4 \pi \tau}\right)+Z_{1}\left(\frac{1}{4 \pi \tau}\right) Z_{1}\left(\frac{3}{4 \pi \tau}\right)\right] \\
& -\frac{3 \pi}{2} Z_{1 / 2}\left(\frac{1}{4 \pi \tau}\right) Z_{3 / 2}\left(\frac{1}{4 \pi \tau}\right)+\frac{\pi}{2} Z_{1 / 2}\left(\frac{1}{4 \pi \tau}\right) \\
& \times Z_{3 / 2}\left(\frac{3}{4 \pi \tau}\right)-\frac{23}{12} \Delta^{2} Z_{1}\left(\frac{1}{4 \pi \tau}\right) \\
a_{3}= & \frac{\pi}{32} Z_{1}\left(\frac{1}{4 \pi \tau}\right) Z_{2}\left(\frac{1}{4 \pi \tau}\right)-\frac{9 \pi}{32} Z_{1}\left(\frac{1}{4 \pi \tau}\right) Z_{2}\left(\frac{3}{4 \pi \tau}\right) \\
& +\frac{45 \pi^{2}}{32}\left[Z_{3 / 2}^{2}\left(\frac{1}{4 \pi \tau}\right)+Z_{1 / 2}\left(\frac{1}{4 \pi \tau}\right) Z_{5 / 2}\left(\frac{1}{4 \pi \tau}\right)\right] \\
& +\frac{9 \pi^{2}}{8}\left[Z_{3 / 2}^{2}\left(\frac{1}{4 \pi \tau}\right)+Z_{1 / 2}\left(\frac{1}{4 \pi \tau}\right) Z_{5 / 2}\left(\frac{1}{4 \pi \tau}\right)\right]
\end{aligned}
$$

Behaviour of Thin Magnetic Films at Low Temperatures

$$
\begin{aligned}
& -\frac{9 \pi}{2} Z_{1}\left(\frac{1}{4 \pi \tau}\right) Z_{2}\left(\frac{1}{4 \pi \tau}\right) \\
& -\frac{15 \pi^{2}}{32}\left[Z_{3 / 2}\left(\frac{1}{4 \pi \tau}\right) Z_{3 / 2}\left(\frac{3}{4 \pi \tau}\right)\right. \\
& \left.+Z_{1 / 2}\left(\frac{1}{4 \pi \tau}\right) Z_{5 / 2}\left(\frac{3}{4 \pi \tau}\right)\right] \\
& -\frac{3 \pi^{2}}{8}\left[Z_{3 / 2}\left(\frac{1}{4 \pi \tau}\right) Z_{3 / 2}\left(\frac{3}{4 \pi \tau}\right)\right. \\
& \left.+Z_{1 / 2}\left(\frac{1}{4 \pi \tau}\right) Z_{5 / 2}\left(\frac{3}{4 \pi \tau}\right)\right] \\
& +\frac{3 \pi}{2} Z_{1}\left(\frac{1}{4 \pi \tau}\right) Z_{1}\left(\frac{3}{4 \pi \tau}\right)-\frac{23}{12} \Delta Z_{1} \Delta Z_{2}+4 \pi^{2} \Delta^{2} Z_{3 / 2}
\end{aligned}
$$

where

$$
\Delta Z_{p}=Z_{p}\left(\frac{1}{4 \pi \tau}\right)-Z_{p}\left(\frac{3}{4 \pi \tau}\right)
$$

For the layer $n_{z}=1$ ordering parameter is given by:

$$
\sigma^{01}(1)=\sigma_{h}^{01}(1)+\delta_{a} \sigma^{01}(1)
$$

where

$$
\begin{aligned}
\sigma_{h}^{01}(1)= & 1-2 \tau Z_{1}\left(\frac{3}{4 \pi \tau}\right)-\pi \tau^{2} Z_{2}\left(\frac{3}{4 \pi \tau}\right) \\
& +O\left(\tau^{3} Z\right)
\end{aligned}
$$

and

$$
\delta_{a} \sigma^{01}(1)=b_{1} \tau+b_{2} \tau^{2}+b_{3} \tau^{3}+O\left(\tau^{4} Z^{2}\right)
$$

where

$$
\begin{aligned}
b_{1}= & \frac{11}{8 \pi} Z_{0}\left(\frac{3}{4 \pi \tau}\right) Z_{1}\left(\frac{1}{4 \pi \tau}\right)+\frac{61}{8 \pi} Z_{0}\left(\frac{3}{4 \pi \tau}\right) Z_{1}\left(\frac{3}{4 \pi \tau}\right) \\
b_{2}= & \frac{11}{16}\left[Z_{0}\left(\frac{3}{4 \pi \tau}\right) Z_{2}\left(\frac{1}{4 \pi \tau}\right)+Z_{1}\left(\frac{1}{4 \pi \tau}\right) Z_{1}\left(\frac{3}{4 \pi \tau}\right)\right] \\
& +\frac{61}{16}\left[Z_{0}\left(\frac{3}{4 \pi \tau}\right) Z_{2}\left(\frac{3}{4 \pi \tau}\right)+Z_{1}^{2}\left(\frac{3}{4 \pi \tau}\right)\right] \\
& +\frac{3 \pi}{2} Z_{1 / 2}\left(\frac{3}{4 \pi \tau}\right) Z_{3 / 2}\left(\frac{3}{4 \pi \tau}\right)+\frac{\pi}{2} Z_{1 / 2}\left(\frac{3}{4 \pi \tau}\right) \\
& \times Z_{3 / 2}\left(\frac{1}{4 \pi \tau}\right)-\frac{23}{12} \Delta^{2} Z_{1}\left(\frac{1}{4 \pi \tau}\right) \\
b_{3}= & \frac{11 \pi}{32} Z_{1}\left(\frac{3}{4 \pi \tau}\right) Z_{2}\left(\frac{1}{4 \pi \tau}\right)+\frac{61 \pi}{32} Z_{1}\left(\frac{3}{4 \pi \tau}\right) Z_{2}\left(\frac{3}{4 \pi \tau}\right) \\
& -\frac{45 \pi^{2}}{32}\left[Z_{3 / 2}^{2}\left(\frac{3}{4 \pi \tau}\right)+Z_{1 / 2}\left(\frac{3}{4 \pi \tau}\right) Z_{5 / 2}\left(\frac{3}{4 \pi \tau}\right)\right] \\
& -\frac{9 \pi^{2}}{8}\left[Z_{3 / 2}^{2}\left(\frac{3}{4 \pi \tau}\right)+Z_{1 / 2}\left(\frac{3}{4 \pi \tau}\right) Z_{5 / 2}\left(\frac{3}{4 \pi \tau}\right)\right] \\
& +\frac{9 \pi}{2} Z_{1}\left(\frac{3}{4 \pi \tau}\right) Z_{2}\left(\frac{3}{4 \pi \tau}\right)
\end{aligned}
$$




$$
\begin{aligned}
& -\frac{15 \pi^{2}}{32}\left[Z_{3 / 2}\left(\frac{3}{4 \pi \tau}\right) Z_{3 / 2}\left(\frac{1}{4 \pi \tau}\right)\right. \\
& \left.+Z_{1 / 2}\left(\frac{3}{4 \pi \tau}\right) Z_{5 / 2}\left(\frac{1}{4 \pi \tau}\right)\right] \\
& -\frac{3 \pi^{2}}{32}\left[Z_{3 / 2}\left(\frac{3}{4 \pi \tau}\right) Z_{3 / 2}\left(\frac{1}{4 \pi \tau}\right)\right. \\
& \left.\quad+Z_{1 / 2}\left(\frac{3}{4 \pi \tau}\right) Z_{5 / 2}\left(\frac{1}{4 \pi \tau}\right)\right] \\
& +\frac{3 \pi}{2} Z_{1}\left(\frac{3}{4 \pi \tau}\right) Z_{2}\left(\frac{1}{4 \pi \tau}\right)-\frac{23}{12} \Delta Z_{1} \Delta Z_{2}+4 \pi^{2} \Delta^{2} Z_{3 / 2}
\end{aligned}
$$

Taking into account the film geometry we immediately can write for subfilm $(1,2)$ :

$$
\begin{aligned}
& \sigma^{12}(1)=\sigma^{01}(1) \\
& \sigma^{12}(2)=\sigma^{01}(0)
\end{aligned}
$$

In subfilm $(0,2)$ the ordering parameters are given by:

$$
\sigma^{10}(0)=\sigma^{02}(2)=\frac{1}{2}\left[\sigma^{10}(0)+\sigma^{01}(1)\right]
$$

In connection with the last two formulas see (77).

Ending the analyses of this section we shall estimate the frames of validity of the formulae (117) and (120). Since the error made by the use of the formula (115) is of the order $\tau^{2} Z^{3}$, while the minimal terms in (117) and (120) are of the order $\tau^{3} Z^{2}$, the formulae (117) and (120) for ordering parameters are correct if $\tau<Z$. This condition is satisfied if $0<\tau<0.02$. For the ferromagnet with transition temperature of the order $T_{C}=1000 \mathrm{~K}$ the formulae (117) and (120) are correct for the values of temperatures lying in the interval $\{0<T<150 \mathrm{~K}\}$.

\section{CONCLUSION}

The results obtained here are heterogeneous and significantly differ form the results for corresponding macroscopic structure. It is useful, therefore, to expose the concluding remarks taxatively:

(1) In macroscopic structure the small quantity in relative magnetization expansion is the relative temperature $\tau=\Theta /(2 \pi I)$. In the corresponding film appear two small quantities: relative temperature $\tau$ and exponentially small quantities given by the Dyson's functions $Z_{p}((2 \lambda-1) /$ $(4 \pi \tau))$. The exponentially small terms appear already in harmonical (Bloch's) approximation (see Section 3). It was reason for the use of exact Agranovich-Toshich Bozon representation of spin operators in these analyses.

(2) In the macroscopic structure the harmonic magnetization contains the terms $\tau^{3 / 2}, \tau^{5 / 2}$ and $\tau^{7 / 2}$, while anharmonic correction is proportional to $\tau^{4}$. The harmonic terms are the consequence of application small wave vector approximation up to $k^{6}$. In the film the harmonic terms are of the order $\tau Z, \tau^{2} Z$ etc., while the anharmonic corrections are of the type $\tau Z^{2}, \tau^{2} Z^{2}$ etc. This fact complicates comparing of the film to the corresponding macroscopic structure. Independently, the following conclusion is evident: the thin magnetic films are more resistant to temperature changes than the corresponding bulk ferromagnet.

(3) Magnetization of thin films depends on configurational index $n_{z}$. This is the consequence of disturbed translational symmetry along $z$-direction.

(4) The more essential consequence of translation symmetry disturbance is appearance of autoreduction in thin film. Namely the transition from configurational space into the momentum one is not isomorphic transformation of the type $N \leftrightarrow N$. In the thin magnetic film we have the transition of the type $N \leftrightarrow$ $N-1$. In the three layer film configurational index $n_{z}$ takes three values: $n_{z}=0, n_{z}=1$ and $n_{z}=2$, while the momentum index $\lambda$ takes two values: $\lambda=1$ and $\lambda=2$. It means that in three layer films the initial excitations appear in two-layer substructure, only. Those substructure are $(0,1),(1,2)$ and $(0,2)$. The approach used does not give the answer to the question: what happens in the "empty" layer? The most probable answer is that in the empty layer appears localized spin excitations. It is important to note that autoreduction can be considered as qualitative difference between nano- and macroscopic structures.

(5) In thin magnetic films magnetization of boundary layers more rapidly decreases with the increase of the temperature than in central layer. It is physically justified since the field of exchange forces on boundaries is weaker than the field of forces acting to spins at the central layer.

(6) The lower magnetization in boundary layers with respect to the magnetization of the central layer means that the concentrations of excitations in boundary layers are higher than those in the central layer. In other words spin waves energy of boundary layers is higher than that of the central layer. This causes energy flow from the places of higher energy to the place of lower energy. This fact is expressed by the formula (78) were the transitions of excitations from the layers $n_{z}=0$ and $n_{z}=2$ to the layer $n_{z}=1$ are different from zero, while the inverse transition are equal to zero.

Acknowledgment: This work was supported by the Serbian Ministry of Science and Technology: Grant No. 141044, and by Vojvodina Academy of Sciences and Arts.

\section{References}

1. F. Bloch, Z. Physik 61, 206 (1930).

2. M. Bloch, Phys. Rev. Lett. 9, 286 (1962).

3. T. Holstein and H. Primakoff, Phys. Rev. 58, 1098 (1940).

4. F. J. Dyson, Phys. Rev. 102, 1217 (1956).

5. F. J. Dyson, Phys. Rev. 102, 1230 (1956).

6. S. V. Maleev, Zh. Eksperim. i Teor. Fiz 33, 1010 (1957). 
7. S. V. Tyablikov, Methods in the Quantum Theory in Magnetism, Plenum Press, New York (1967).

8. P. A. Kullstam, IEEE Transactions on Education 34, 155 (1991).

9. B. S. Tošić, Statistical Physics, Faculty of Science, Novi Sad (1978).

10. R. A. Tahir-Kheli and D. Ter Haar, Phys. Rev. 127, 95 (1962).

11. R. A. Tahir-Kheli, Phys. Rev. 132, 689 (1963).

12. R. A. Tahir-Kheli and H. B. Callen, Phys. Rev. 135, A679 (1964).

13. R. A Tahir-Kheli and D. Ter Haar, Phys. Rev. 130, 108 (1963).

14. H. B. Callen, Phys. Lett. 4, 161 (1963).

15. N. N. Bogolubov and S. V. Tyablikov, Dokl. Akadem. Nauk USSR 126, 53 (1959)

16. H. A. Brown, Phys. Rev. 104, 624 (1956).

17. H. A. Brown and J. M. Luttinger, Phys.Rev. 100, 685 (1955).

18. V. M. Agranovich and B. S. Toshich, JETP 53, 149 (1967).

19. M. L. Kulić and B. S. Tošić, Phys. Stat. Sol. (b) 56, K79 (1973).

20. Group of authors, Jubilee of Bratislav Tošić, SFIN 1 (1996).

21. V. Antonov, Electronic Structure and Magneto-Optical Properties of Solids, Kluwer Academic Publishers (2004).

22. K. H. J. Buschow and F. R. De Boer, Physics of Magnetism and Magnetic Materials, Kluwer, New York, Boston (2003).

23. M. Getzlaff, Fundamentals of Magnetism, Springer, Berlin, Heidelberg, New York (2008).
24. J. Stöhr and H. C. Siegmann, Magnetism From Fundamentals to Nanoscale Dynamics, Springer, Berlin, Heidelberg (2006).

25. R. M. White, Quantum Theory of Magnetism, Magnetic Properties of Materials, Springer, Berlin, Heidelberg (2007).

26. S. Maekawa and T. Shinjo, Spin Dependent Transport in Magnetic Nanostructures, CRC Boca Raton, London, New York, Washington, D.C. (2002).

27. M. Pavkov, M. Škrinjar, D. Kapor, M. Pantić, and S. Stojanović, Phys. Rev. B 65, 104512 (2002).

28. M. Manojlović, M. Pavkov, M. Škrinjar, M. Pantić, D. Kapor, and S. Stojanović, Phys. Rev. B 71, 132510 (2005).

29. P. Fröbrich and P. J. Kuntz, Physics Reports 432, 223 (2006)

30. R. Radu, M. Etzkorn, R. Siebrecht et al., Phys. Rev. B 67, 134409 (2003).

31. V. Sajfert, R. Đajić, M. Ćetković, and B. Tošić, Nanotechnology 14, 358 (2003).

32. V. Sajfert, R. Đajić, and B. S. Tošić, J. Nanosci. Nanotechnol. 4, 886 (2004).

33. V. Sajfert, J. Šetrajčić, B. Tošić, and R. Đajić, Czechoslovak Journal of Physics 54, 975 (2004).

34. V. Sajfert, J. P. Šetrajčić, S. Jaćimovski, and B. Tošić, Physica E 25, 479 (2005).

35. V. Sajfert, J. Šetrajčić, D. Popov, and B. Tošić, Physica A 353C, 217 (2005).

Received: 10 November 2008. Accepted: 28 November 2008. 